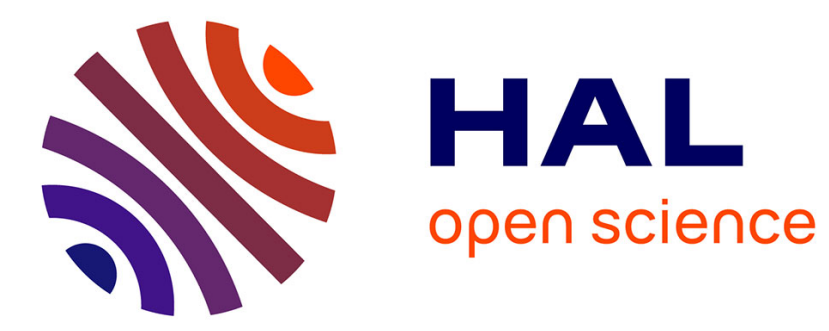

\title{
Low scale separation induces modification of apparent solute transport regime in porous media \\ Pascale Royer
}

\section{To cite this version:}

Pascale Royer. Low scale separation induces modification of apparent solute transport regime in porous media. Mechanics Research Communications, 2018, 87, pp.29-34. 10.1016/j.mechrescom.2017.12.003 . hal-01667675

\section{HAL Id: hal-01667675 \\ https://hal.umontpellier.fr/hal-01667675}

Submitted on 19 Dec 2017

HAL is a multi-disciplinary open access archive for the deposit and dissemination of scientific research documents, whether they are published or not. The documents may come from teaching and research institutions in France or abroad, or from public or private research centers.
L'archive ouverte pluridisciplinaire $\mathbf{H A L}$, est destinée au dépôt et à la diffusion de documents scientifiques de niveau recherche, publiés ou non, émanant des établissements d'enseignement et de recherche français ou étrangers, des laboratoires publics ou privés. 


\title{
Low scale separation induces modification of apparent solute transport regime in porous media
}

\author{
PAscale RoYeR* \\ Laboratoire de Mécanique et Génie Civil - LMGC - (Univ. Montpellier, CNRS) \\ pascale.royer@umontpellier.fr
}

December 13, 2017

\begin{abstract}
First order asymptotic homogenization allows to determine the effective behaviour of a porous medium by starting from the pore scale description, when there is a large separation between the pore scale and the macroscopic scale. When the scale ratio is âĂIJsmall but not too small, $\hat{a} \breve{A} I$ additional terms need to be taken into account, which can be obtained by exploiting higher order equations in the asymptotic homogenization procedure. The aim of the present study is to derive second order models to describe solute transport in a macroscopically homogeneous porous medium at low scale separation. The three following macroscopic transport regimes are successively considered: pure diffusion with fluid at rest, predominant diffusion with fluid in motion and advection-diffusion. The results show that while the transport regime remains of diffusive type when the fluid is at rest, low scale separation induces modification of apparent transport regime when fluid is in motion. Indeed, predominant diffusion and advection-diffusion lead to the apparent regimes of advection-diffusion and of dispersion, respectively.
\end{abstract}

\section{INTRODUCTION}

Most studies in the theory of flow and transport in porous media are based on the exploitation of the continuum theory, which requires that the condition of separation of scales be fulfilled. First order asymptotic homogenization can be used to derive such continuum models. When the scale ratio is "small but not too small", the additional terms which are required to account for micro-structural scale effects can be obtained by considering higher order correctors in the asymptotic homogenization procedure. The present paper is aimed at analysing the influence of low scale separation on solute transport in macroscopically homogeneous rigid porous media. The objective is to derive second order homogenized models for the three following macroscopic transport regimes: pure diffusion with the fluid at rest, predominant diffusion with the fluid in motion and advection-diffusion. The results show that low scale separation induces modification of apparent transport regime when fluid is in motion. The paper is organised as follows. Section II presents a brief description of the homogenization methodology. Second order homogenization of pure diffusion is detailed in Section III. and second order homogenization of convection-diffusion in the regimes of predominant diffusion and of advection diffusion is the purpose of Section IV. Finally, Section $\mathrm{V}$ presents a summary of the main theoretical results contained in this work and highlights conclusive remarks. 


\section{HoMOgENIZATION METHOD}

\section{i. Medium under consideration}

We consider a periodic porous medium, of macroscopic characteristic size $L$, and made of a solid matrix and of a fluid-saturated pore space. We further denote the periodic cell by $\Omega$, its characteristic length by $l$, and we formulate the condition of separation of scales by $\varepsilon=l / L \ll 1$. Within the periodic cell, we denote by $\Omega_{\mathrm{p}}$ the fluid-saturated pore domain, by $\Omega_{\mathrm{s}}$ the solid matrix, and by $\Gamma$ their common interface, as depicted in Fig. 1 . Using the two characteristic lengths, $l$ and $L$, and the physical space variable, $\vec{X}$, we define two dimensionless space variables: $\vec{y}=\vec{X} / l, \vec{x}=\vec{X} / L$, and $\vec{y}$ and $\vec{x}$ describe variations on the microscopic and the macroscopic scales, respectively. Invoking the differentiation rule of multiple variables, the gradient operator with respect to $\vec{X}$ is written as

$$
\vec{\nabla}_{X}=\frac{1}{l} \vec{\nabla}_{y}+\frac{1}{L} \vec{\nabla}_{x}
$$

\section{ii. Nondimensionalisation of pore scale description}

The methodology firstly consists in writing the dimensionless pore scale description, which is the set of dimensionless equations that describe the phenomena being considered within the periodic unit cell. Each quantity in a dimensionless equation is the ratio of its physical counterpart to its characteristic value (indexed by c). This writing gives rise to dimensionless numbers, which are defined by means of characteristic values. These dimensionless parameters are then estimated with respect to powers of the small parameter $\varepsilon$. A parameter $\mathbb{P}$ is said to be of order $\varepsilon^{p}, \mathbb{P}=\mathcal{O}\left(\varepsilon^{p}\right)$, when

$$
\varepsilon^{p+1} \ll \mathbb{P} \ll \varepsilon^{p-1} .
$$

The dimensionless writing of the equations requires the choice of a characteristic length for the dimensionless writing of space derivatives. We arbitrarily choose $L$ as the reference characteristic length. The dimensionless gradient operator is thus $L \vec{\nabla}_{X}$, which by Eq. (1) is given by

$$
\vec{\nabla}=L \vec{\nabla}_{X}=\varepsilon^{-1} \vec{\nabla}_{y}+\vec{\nabla}_{x} .
$$

\section{iii. Homogenisation procedure}

The homogenization method being used is an asymptotic approach. It is therefore based upon the fundamental assumption that the unknown fields can be written in the form of asymptotic expansions in powers of $\varepsilon$

$$
\psi=\psi^{0}(\vec{y}, \vec{x})+\varepsilon \psi^{1}(\vec{y}, \vec{x})+\varepsilon^{2} \psi^{2}(\vec{y}, \vec{x})+\ldots
$$

in which functions $\psi^{i}$ are $\Omega$-periodic in variable $\vec{y}$. The method consists in incorporating the asymptotic expansions in the dimensionless local description, while replacing the dimensionless numbers by their orders of magnitude in power of $\varepsilon$ and taking into account the expression of the dimensionless gradient operator Eq. (3). This leads to approximated governing equations and boundary conditions at the successive orders, which together with the condition of periodicity define well posed boundary value problems within the periodic unit cell, from which functions $\psi^{i}$ can be determined. Existence of solutions requires that volume averaged equations be satisfied. These latter actually describe the macroscopic behaviour at successive orders.

\section{SECOND ORDER HOMOGENIZATION OF PURE DIFFUSION}

Considering the fluid-saturated medium depicted in Fig. 1, let us assume that the fluid phase which contains a low concentration of solute, is at rest, so that the solute is only transported by diffusion.

\section{i. Pore scale dimensionless descrip- tion}

The transport of solute by diffusion in the pore domain is described by conservation of mass within $\Omega_{\mathrm{p}}$ and the no-flux boundary condition over $\Gamma$. When cast in dimensionless form, these 


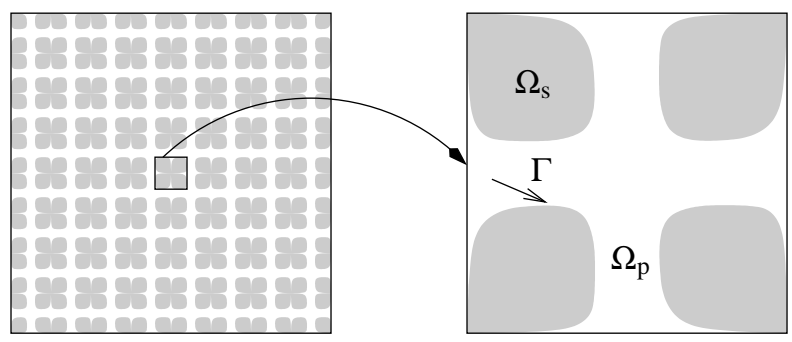

(a)

(b)

Figure 1: Porous medium: (a) Macroscopic sample; (b) Periodic unit cell.

equations read

$$
\begin{cases}\mathbb{N} \frac{\partial c}{\partial t}-\vec{\nabla} \cdot\left(D_{0} \vec{\nabla} c\right)=0 & \text { within } \Omega_{\mathrm{p}}(5) \\ (D \vec{\nabla} c) \cdot \vec{n}=0 & \text { over } \Gamma\end{cases}
$$

where $c$ denotes the solute concentration (mass of solute per unit volume of fluid), $\mathrm{t}$ is time, $D_{0}$ denotes the coefficient of molecular diffusion and $\vec{n}$ represents the unit vector giving the normal to $\Gamma$ exterior to $\Omega_{\mathrm{p}}$. Parameter $\mathbb{N}$ is a dimensionless number, which is defined by

$$
\mathbb{N}=\frac{L^{2}}{D_{0_{\mathrm{c}}} t_{\mathrm{c}}}=\frac{t^{\mathrm{diff}}}{t_{\mathrm{c}}}, \quad t^{\mathrm{diff}}=\frac{L^{2}}{D_{0_{\mathrm{c}}}},
$$

where $t_{c}$ is the characteristic time of observation and $t^{\text {diff }}$ is the characteristic time of diffusion. We take $t_{\mathrm{c}}=t^{\mathrm{diff}}$, i.e.

$$
\mathbb{N}=\mathcal{O}\left(\varepsilon^{0}\right),
$$

which fixes the time of observation so as to get a transient regime on the macroscopic scale.

\section{ii. Second order homogenization}

We consider Eq.(5) with estimate Eq. (8), and boundary condition Eq. (6), and we look for solutions in the form of Eq. (4) for $c$. Incorporating the asymptotic expansion of $c$ and the expression of the dimensionless gradient operator Eq. (3) into Eqs.(5)-(6), and then identifying terms of same power of $\varepsilon$, leads to boundary value problems in terms of functions $c^{i}$ at the successive orders.

\section{Boundary value problem for $c^{0}$}

Considering Eq. (5) at $\mathcal{O}\left(\varepsilon^{-2}\right)$ and Eq. (6) at $\mathcal{O}\left(\varepsilon^{-1}\right)$, we get the first order boundary value problem

$$
\left\{\begin{array}{l}
\frac{\partial}{\partial y_{i}}\left(D_{0} \frac{\partial c^{0}}{\partial y_{i}}\right)=0 \quad \text { within } \Omega_{\mathrm{p}} \\
D_{0} \frac{\partial c^{0}}{\partial y_{i}} n_{i}=0 \quad \text { over } \Gamma \\
c^{0}: \text { periodic in } \vec{y},
\end{array}\right.
$$

from which it is clear that the concentration $c^{0}$ is constant over the period

$$
c^{0}=c^{0}(\vec{x}, t)
$$

\section{Boundary value problem for $c^{1}$}

At the second order, we obtain the following boundary value problem for $c^{1}$ :

$$
\left\{\begin{array}{l}
\frac{\partial}{\partial y_{i}}\left[D_{0}\left(\frac{\partial c^{1}}{\partial y_{i}}+\frac{\partial c^{0}}{\partial x_{i}}\right)\right]=0 \text { within } \Omega_{\mathrm{p}(13)} \\
{\left[D_{0}\left(\frac{\partial c^{1}}{\partial y_{i}}+\frac{\partial c^{0}}{\partial x_{i}}\right)\right] n_{i}=0} \\
c^{0}: \text { periodic in } \vec{y}
\end{array}\right.
$$

By virtue of linearity, the solution reads

$$
c^{1}=\chi_{j}(\vec{y}) \frac{\partial c^{0}}{\partial x_{j}}+\bar{c}^{1}(\vec{x}, t),
$$

where $\bar{c}^{1}(\vec{x}, t)$ is an arbitrary function. Note that, to render the solution unique, we impose 
that $\vec{\chi}$ is average to zero $\langle\vec{\chi}>=\overrightarrow{0}$ (e.g. Bensoussan et al. (1978), Sanchez-Palencia (1980)), where the volume mean over $\Omega_{p}$ is defined by

$$
<\cdot>=\frac{1}{|\Omega|} \int_{\Omega_{\mathrm{p}}} \cdot d \Omega
$$

Note further that, since we are considering a macroscopically homogeneous medium, $\vec{\chi}$ doesn't depend on variable $\vec{x}: \vec{\chi}=\vec{\chi}(\vec{y})$.

\section{First order macroscopic description}

Let consider the boundary value problem Eqs. (5)-(6) at the third order:

$$
\left\{\begin{array}{l}
\frac{\partial c^{0}}{\partial t}-\frac{\partial}{\partial y_{i}}\left[D_{0}\left(\frac{\partial c^{2}}{\partial y_{i}}+\frac{\partial c^{1}}{\partial x_{i}}\right)\right]- \\
\frac{\partial}{\partial x_{i}}\left[D_{0}\left(\frac{\partial c^{1}}{\partial y_{i}}+\frac{\partial c^{0}}{\partial x_{i}}\right)\right]=0 \text { within } \Omega_{\mathrm{p}} \\
{\left[D_{0}\left(\frac{\partial c^{2}}{\partial y_{i}}+\frac{\partial c^{1}}{\partial x_{i}}\right)\right] n_{i}=0 \quad \text { over } \Gamma}
\end{array}\right.
$$

The homogenization procedure consists now in integrating Eq. (18) over $\Omega_{\mathrm{p}}$. This leads to the so called compatibility condition, which is a necessary and sufficient condition for the existence of solutions, and further represents the first order macroscopic description. Integrating equation Eq. (18) and invoking Gauss' theorem, while taking boundary condition Eq. (19) into account, together with the condition of periodicity, yields

$$
\phi \frac{\partial c^{0}}{\partial t}-\frac{\partial}{\partial x_{i}}\left(<D_{0}\left(\frac{\partial c^{1}}{\partial y_{i}}+\frac{\partial c^{0}}{\partial x_{i}}>\right)=0 .\right.
$$

Now, using Eq. (16), we can write:

$$
\frac{\partial c^{1}}{\partial y_{i}}+\frac{\partial c^{0}}{\partial x_{i}}=\gamma_{i j}^{0} \frac{\partial c^{0}}{\partial x_{j}}, \quad \gamma_{i j}^{0}=\frac{\partial \chi_{j}}{\partial y_{i}}+I_{i j} .
$$

Eq. (20) can thus be rewritten as follows

$$
\phi \frac{\partial c^{0}}{\partial t}-\frac{\partial}{\partial x_{i}}\left(D_{i j} \frac{\partial c^{0}}{\partial x_{j}}\right)=0,
$$

where

$$
D_{i j}=\frac{1}{|\Omega|} \int_{\Omega_{\mathrm{p}}} D_{0} \gamma_{i j}^{0} d \Omega
$$

is the tensor of effective diffusion. Eq. (22) may be expressed as

$$
\left\{\begin{array}{l}
\phi \frac{\partial<c>}{\partial t}-\frac{\partial}{\partial x_{i}}\left(D_{i j} \frac{\partial<c>}{\partial x_{j}}\right) \\
=\mathcal{O}\left(\varepsilon \phi \frac{\partial<c>}{\partial t}\right) .
\end{array}\right.
$$

In the above writing $\langle c\rangle$ represents the first order average concentration, where the first order average of any field $\psi$ is defined by

$$
<\psi>=<\psi^{0}>+\mathcal{O}(\varepsilon<\psi>) .
$$

The above equation represents the first order macroscopic description for pure diffusion.

\section{Boundary value problem for $c^{2}$}

The third order boundary value problem Eqs. (18)-(19) can be transformed so as to obtain the following well-posed problem for $c^{2}$

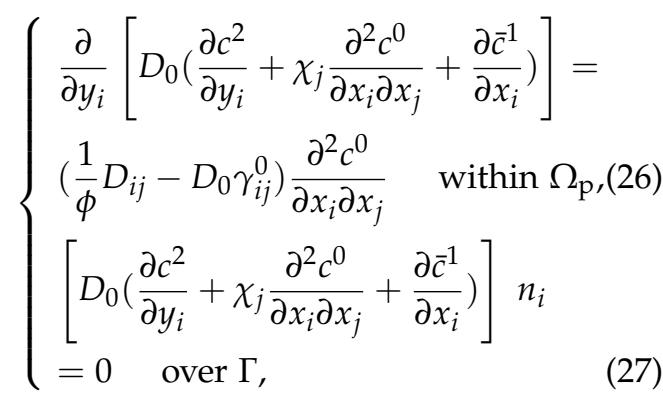

whose solution reads

$$
c^{2}=\eta_{j k}(\vec{y}) \frac{\partial^{2} c^{0}}{\partial x_{j} \partial x_{k}}+\chi_{j}(\vec{y}) \frac{\partial \bar{c}^{1}}{\partial x_{j}}+\bar{c}^{2}(\vec{x}, t),
$$

where $\bar{c}^{2}(\vec{x}, t)$ is an arbitrary function and where $\left\langle\eta_{j k}>=0\right.$.

\section{Derivation of the second order corrector}

The fourth order of Eqs. (5)-6) yields:

$$
\left\{\begin{array}{l}
\frac{\partial c^{1}}{\partial t}-\frac{\partial}{\partial y_{i}}\left[D_{0}\left(\frac{\partial c^{3}}{\partial y_{i}}+\frac{\partial c^{2}}{\partial x_{i}}\right)\right]- \\
\frac{\partial}{\partial x_{i}}\left[D_{0}\left(\frac{\partial c^{2}}{\partial y_{i}}+\frac{\partial c^{1}}{\partial x_{i}}\right)\right]=0 \text { within } \Omega_{p}(29) \\
{\left[D_{0}\left(\frac{\partial c^{3}}{\partial y_{i}}+\frac{\partial c^{2}}{\partial x_{i}}\right)\right] n_{i}=0 \quad \text { over } \Gamma . \quad(30)}
\end{array}\right.
$$


The second order corrector of the macroscopic description is obtained by integrating Eq. (29) over $\Omega_{p}$. This leads to

$$
\phi \frac{\partial \bar{c}^{1}}{\partial t}-\frac{\partial}{\partial x_{i}}\left(E_{i j k} \frac{\partial^{2} c^{0}}{\partial x_{j} \partial x_{k}}+D_{i j} \frac{\partial \bar{c}^{1}}{\partial x_{j}}\right)=0,
$$

where

$$
E_{i j k}=<D_{0}\left(\frac{\partial \eta_{j k}}{\partial y_{i}}+\chi_{i} I_{j k}\right)>.
$$

The third order tensor $E_{i j k}$ is symmetric with respect to its last two indices and antisymmetric with respect to its first two indices, and, as a result of this anti-symmetry property, the second order gradient term of Eq. (31) vanishes ( $C f$. A). Thus, the second order corrector finally reads:

$$
\phi \frac{\partial \bar{c}^{1}}{\partial t}-\frac{\partial}{\partial x_{i}}\left(D_{i j} \frac{\partial \bar{c}^{1}}{\partial x_{j}}\right)=0 .
$$

\section{Second order macroscopic description}

Let add Eq. (22) to Eq. (33) multiplied by $\varepsilon$. We get

$$
\left\{\begin{array}{l}
\phi \frac{\partial<c>}{\partial t}-\frac{\partial}{\partial x_{i}}\left[D_{i j} \frac{\partial<c>}{\partial x_{j}}\right] \\
=\mathcal{O}\left(\varepsilon^{2} \phi \frac{\partial<c>}{\partial t}\right)
\end{array}\right.
$$

In the above writing, $\langle c\rangle$ represents the second order mean concentration, where, the second order mean of any fied $\psi$ is defined by

$$
<\psi>=<\psi^{0}>+\varepsilon<\psi^{1}>+\mathcal{O}\left(\varepsilon^{2}<\psi>\right) \text {. }
$$

Therefore, the second order macroscopic description of pure diffusion is identical to the first order model, but with a precision $\mathcal{O}\left(\varepsilon^{2}\right)$.

\section{SECOND ORDER HOMOGENIZATION OF CONVECTION DIFFUSION}

i. Pore scale dimensionless description

When the fluid is in motion, the solute is transported by diffusion and convection. Then, the dimensionless transport equations on the pore scale read

$$
\left\{\begin{array}{l}
\mathbb{N} \frac{\partial c}{\partial t}-\vec{\nabla} \cdot\left(D_{0} \vec{\nabla} c-\mathbb{P e} c \vec{v}\right)=0 \text { in } \Omega_{\beta}(36) \\
\vec{v}=\overrightarrow{0} \quad \text { over } \Gamma \\
\left(D_{0} \vec{\nabla} c\right) \cdot \vec{n}=0 \quad \text { over } \Gamma
\end{array}\right.
$$

where $\mathbb{P} e=v_{\mathrm{c}} L / D_{\mathrm{c}}$ denotes the Péclet number. Considering the slow flow of a Newtonian incompressible fluid, its motion is described by Stokes equations

$$
\left\{\begin{array}{l}
\mathbb{F} \mu \Delta \vec{v}-\vec{\nabla} p=\overrightarrow{0} \quad \text { within } \Omega_{\mathrm{p}} \\
\vec{\nabla} \cdot \vec{v}=0 \quad \text { within } \Omega_{\mathrm{p}},
\end{array}\right.
$$

where $\mathbb{F}=\mu_{\mathrm{c}} v_{\mathrm{c}} / L p_{\mathrm{c}}=\mathcal{O}\left(\varepsilon^{2}\right)$ (e.g. Auriault et al. (2005)). Homogenization of the above equations leads to the following macroscopic transport regime accordingly to the order of magnitude of $\mathbb{P} e$ Auriault and Adler (1995):

$$
\begin{cases}\text { diffusion } & \text { when } \mathbb{P} e \leq \mathcal{O}(\varepsilon) \\ \text { advection-diffusion } & \text { when } \mathbb{P} e=\mathcal{O}\left(\varepsilon^{0}\right) \\ \text { advection-dispersion } & \text { when } \mathbb{P} e=\mathcal{O}\left(\varepsilon^{-1}\right)\end{cases}
$$

Whilst the first two models are first order models, the dispersive model requires to account for the second order corrector. Our purpose here is to derive the second order models for $\mathbb{P} e=\mathcal{O}(\varepsilon)$ and $\mathbb{P} e=\mathcal{O}\left(\varepsilon^{0}\right)$.

\section{ii. Second order homogenization of fluid flow}

Homogenization of the fluid flow equations has been performed up to the third order in Auriault et al. (2005). For a macroscopically homogeneous medium, the results at the first two orders can be summarised as follows

$$
\left\{\begin{array}{l}
\frac{\partial}{\partial x_{i}}\left(<v_{i}^{n}>\right)=0,(n=1,2) \\
<v_{i}^{0}>=-\frac{K_{i j}}{\mu} \frac{\partial<p^{0}>}{\partial x_{j}} \\
<v_{i}^{1}>=-\frac{N_{i j k}}{\mu} \frac{\partial^{2}<p^{0}>}{\partial x_{j} \partial x_{k}}-\frac{K_{i j}}{\mu} \frac{\partial<p^{1}>}{\partial x_{j}}
\end{array}\right.
$$


iii. Second order homogenization of convection diffusion at $\mathbb{P e}=\mathcal{O}(\varepsilon)$

\section{First order macroscopic description}

We consider $\mathbb{P} e=\mathcal{O}(\varepsilon)$ and $\mathbb{N}=\mathcal{O}\left(\varepsilon^{0}\right)$ in Eq. (36). At the first two orders, we get the same boundary value problems as those obtained in Section III. and which lead to Eq. (12) and Eq. (16), for $c^{0}$ and $c^{1}$, respectively. At the third order, Eq. (36) yields

$$
\left\{\begin{array}{l}
\frac{\partial c^{0}}{\partial t}-\frac{\partial}{\partial y_{i}}\left[D_{0}\left(\frac{\partial c^{2}}{\partial y_{i}}+\frac{\partial c^{1}}{\partial x_{i}}\right)-c^{0} \vec{v}^{0}\right] \\
-\frac{\partial}{\partial x_{i}}\left[D_{0}\left(\frac{\partial c^{1}}{\partial y_{i}}+\frac{\partial c^{0}}{\partial x_{i}}\right)\right]=0 .
\end{array}\right.
$$

Considering Eq. (40) at the first order

$$
\vec{\nabla}_{y} \cdot \vec{v}^{0}=0,
$$

and Eq. (12), Eq. (42) reduces to Eq. (18). Therefore, its integration leads to Eq. (22) and the first order macroscopic description is the model of diffusion given by Eq. (24). Furthermore, $c^{2}$ is given by Eq. 28).

\section{Second order macroscopic description}

Eq. (36) at the fourth order gives

$$
\left\{\begin{array}{l}
\frac{\partial c^{1}}{\partial t}-\frac{\partial}{\partial y_{i}}\left[D_{0}\left(\frac{\partial c^{3}}{\partial y_{i}}+\frac{\partial c^{2}}{\partial x_{i}}\right)-c^{0} \vec{v}^{1}-c^{1} \vec{v}^{0}\right] \\
-\frac{\partial}{\partial x_{i}}\left[D_{0}\left(\frac{\partial c^{2}}{\partial y_{i}}+\frac{\partial c^{1}}{\partial x_{i}}\right)-c^{0} \vec{v}^{0}\right]=0
\end{array}\right.
$$

and its integration over the period yields

$$
\phi \frac{\partial \bar{c}^{1}}{\partial t}-\frac{\partial}{\partial x_{i}}\left(D_{i j} \frac{\partial \bar{c}^{1}}{\partial x_{j}}\right)+<v_{i}^{0}>\frac{\partial c^{0}}{\partial x_{i}}=0 .
$$

By adding Eq. (22) to Eq. (45) multiplied by $\varepsilon$, we get

$$
\left\{\begin{array}{l}
\phi \frac{\partial<c>}{\partial t}-\frac{\partial}{\partial x_{i}}\left[D_{i j} \frac{\partial<c>}{\partial x_{j}}\right] \\
+\varepsilon<v_{i}>\frac{\partial<c>}{\partial x_{i}}=\mathcal{O}\left(\varepsilon^{2} \phi \frac{\partial<c>}{\partial t}(46)\right.
\end{array}\right.
$$

where $\langle c\rangle$ is the second order average concentration, while $\left\langle v_{i}\right\rangle$ is the first order fluid average velocity and verifies

$$
\left\{\begin{array}{l}
<v_{i}>=-\frac{K_{i j}}{\mu} \frac{\partial<p>}{\partial x_{j}}+\mathcal{O}\left(\varepsilon<v_{i}>(47)\right. \\
\frac{\partial<v_{i}>}{\partial x_{i}}=\mathcal{O}\left(\varepsilon \frac{\partial<v_{i}>}{\partial x_{i}}\right) .
\end{array}\right.
$$

The second order model is therefore the model of advection diffusion, with a precision $\mathcal{O}\left(\varepsilon^{2}\right)$ and in which the velocity is the first order velocity and verifies Darcy's law.

\section{iv. Second order homogenization of convection diffusion at $\mathbb{P} e=\mathcal{O}\left(\varepsilon^{0}\right)$}

\section{First order macroscopic description}

We now consider Eq. 36 with $\mathbb{P} e=\mathcal{O}\left(\varepsilon^{0}\right)$ and $\mathbb{N}=\mathcal{O}\left(\varepsilon^{0}\right)$. The first order boundary value problem is identical to Eqs. (9)-(10), and by Eq. (43), Eqs. (36)-(38) at the second order reduce to Eqs. (13)-(14). Consequently, $c^{0}$ and $c^{1}$ verify Eq. 12) and Eq. 16, respectively. At the third order, Eq. (36) reads

$$
\left\{\begin{array}{l}
\frac{\partial c^{0}}{\partial t}-\frac{\partial}{\partial y_{i}}\left[D_{0}\left(\frac{\partial c^{2}}{\partial y_{i}}+\frac{\partial c^{1}}{\partial x_{i}}\right)-c^{0} v_{i}^{1}-c^{1} v_{i}^{0}\right] \\
-\frac{\partial}{\partial x_{i}}\left[D_{0}\left(\frac{\partial c^{1}}{\partial y_{i}}+\frac{\partial c^{0}}{\partial x_{i}}\right)-c^{0} v_{i}^{0}\right]=0
\end{array}\right.
$$

and the associated boundary conditions are Eq. 19 and: $v_{i}^{0}=v_{i}^{1}=0$ over $\Gamma$. Let us now integrate Eq. (49), so as to obtain the first order homogenized description. Applying the divergence theorem and then using the boundary conditions and the periodicity, we end up with

$$
\phi \frac{\partial c^{0}}{\partial t}-\frac{\partial}{\partial x_{i}}\left(D_{i j} \frac{\partial c^{0}}{\partial x_{j}}\right)+<v_{i}^{0}>\frac{\partial c^{0}}{\partial x_{i}}=0 .
$$

Therefore, the first order macroscopic description is the model of advection-diffusion and reads

$$
\left\{\begin{array}{l}
\phi \frac{\partial<c>}{\partial t}-\frac{\partial}{\partial x_{i}}\left[D_{i j} \frac{\partial<c>}{\partial x_{j}}\right] \\
+<v_{i}>\frac{\partial<c>}{\partial x_{i}}=\mathcal{O}\left(\varepsilon \phi \frac{\partial<c>}{\partial t}\right)(
\end{array}\right.
$$

in which $\langle c\rangle$ is the first order mean concentration. 


\section{Second order macroscopic description}

The third order equation Eq. (49), can be transformed to obtain a formulation for determining $c^{2}$ (Cf. Appendix B):

$$
\left\{\begin{array}{l}
\frac{\partial}{\partial y_{i}}\left[D_{0}\left(\frac{\partial c^{2}}{\partial y_{i}}+\chi_{j} \frac{\partial^{2} c^{0}}{\partial x_{i} \partial x_{j}}+\frac{\partial \bar{c}^{1}}{\partial x_{i}}\right)\right]= \\
\left(\frac{1}{\phi} D_{i j}-D_{0} \gamma_{i j}^{0}\right) \frac{\partial^{2} c^{0}}{\partial x_{i} \partial x_{j}} \\
+\left(v_{i}^{0} \gamma_{i j}^{0}-\frac{1}{\phi}<v_{j}^{0}>\right) \frac{\partial c^{0}}{\partial x_{j}} .
\end{array}\right.
$$

The above equation with the associated boundary condition, given by Eq. 27), constitute a well-posed boundary value problem for $c^{2}$, whose solution reads

$$
\left\{\begin{array}{l}
c^{2}=\eta_{j k}(\vec{y}) \frac{\partial^{2} c^{0}}{\partial x_{j} \partial x_{k}}+\pi_{j}(\vec{y}) \frac{\partial c^{0}}{\partial x_{j}} \\
+\chi_{j}(\vec{y}) \frac{\partial \bar{c}^{1}}{\partial x_{j}}+\bar{c}^{2}(\vec{x}, t)
\end{array}\right.
$$

where $\bar{c}^{2}(\vec{x}, t)$ is an arbitrary function and where $\left\langle\eta_{j k}>=0,<\pi_{j}>=0\right.$. Note that by definition, vector $\vec{\pi}$ depends on the fluid velocity. At the fourth order, equation Eq. (36) yields

$$
\left\{\begin{array}{l}
\frac{\partial c^{1}}{\partial t}-\frac{\partial}{\partial y_{i}}\left[D_{0}\left(\frac{\partial c^{3}}{\partial y_{i}}+\frac{\partial c^{2}}{\partial x_{i}}\right)\right] \\
+\frac{\partial}{\partial y_{i}}\left[c^{0} v_{i}^{2}-c^{1} v_{i}^{1}-c^{2} v_{i}^{0}\right] \\
-\frac{\partial}{\partial x_{i}}\left[D_{0}\left(\frac{\partial c^{2}}{\partial y_{i}}+\frac{\partial c^{1}}{\partial x_{i}}\right)-c^{0} v_{i}^{1}-c^{1} v_{i}^{0}\right]=0
\end{array}\right.
$$

and the associated boundary conditions are Eq. (30) and: $v_{i}^{0}=v_{i}^{1}=v_{i}^{2}=0$ over $\Gamma$. The secondorder corrector of the macroscopic description is obtained by integrating Eq. (54) over $\Omega_{p}$. This leads to

$$
\left\{\begin{array}{l}
\phi \frac{\partial \bar{c}^{1}}{\partial t}-\frac{\partial}{\partial x_{i}}\left(D_{i j}^{\prime} \frac{\partial c^{0}}{\partial x_{j}}+D_{i j} \frac{\partial \bar{c}^{1}}{\partial x_{j}}\right) \\
+<v_{i}^{1}>\frac{\partial c^{0}}{\partial x_{i}}+<v_{i}^{0}>\frac{\partial \bar{c}^{1}}{\partial x_{i}}=0,
\end{array}\right.
$$

where

$$
D_{i j}^{\prime}=<D_{0} \frac{\partial \pi_{j}}{\partial y_{i}}-v_{i}^{0} \chi_{j}>.
$$

From its definition Eq. (56), we see that the second order tensor $D_{i j}^{\prime}$ contains a convective term: it is therefore a dispersion tensor. Let us add Eq. (22), to Eq. (55) multiplied by $\varepsilon$. We get:

$$
\left\{\begin{array}{c}
\phi \frac{\partial<c>}{\partial t}-\frac{\partial}{\partial x_{i}}\left[\left(D_{i j}+\varepsilon D_{i j}^{\prime}\right) \frac{\partial<c>}{\partial x_{j}}\right] \\
+<v_{i}>\frac{\partial<c>}{\partial x_{i}}=\mathcal{O}\left(\varepsilon^{2} \phi \frac{\partial<c>}{\partial t}\right) .(57)
\end{array}\right.
$$

In the above equation, $\left\langle v_{i}\right\rangle$ is the second order fluid velocity, given by

$$
\left\{\begin{array}{l}
<v_{i}>=-\frac{N_{i j k}}{\mu} \frac{\partial^{2}<p>}{\partial X_{j} \partial_{k}}-\frac{K_{i j}}{\mu} \frac{\partial<p>}{\partial X_{j}} \\
+\mathcal{O}\left(\varepsilon^{2}<v_{i}>\right), \\
\frac{\partial<v_{i}>}{\partial X_{i}}=\mathcal{O}\left(\varepsilon^{2} \frac{\partial<v_{i}>}{\partial X_{i}}\right) .
\end{array}\right.
$$

Therefore, the second order macroscopic transport description is a model of advectiondispersion, in which the fluid velocity verifies a second order law Eq.(58).

\section{Conclusion}

In the present paper, second order asymptotic homogenization is performed for three regimes of solute transport in macroscopically homogeneous porous media. When the fluid is at rest, the second order model remains of diffusive type. This shows that low scale separation has no impact on the apparent transport regime, and furthermore highlights the robustness of the model of effective diffusion, when the fluid is at rest. When the fluid is in motion and at $\mathbb{P e}=\mathcal{O}(\varepsilon)$, we get the model of effective diffusion at the first order, and the model of advection-diffusion at the second order. This result can be generalised to lower orders of the Péclet number: at $\mathbb{P e}=\mathcal{O}\left(\varepsilon^{p}\right)$ with $p \geq 1$, the apparent advective-diffusion regime occurs at order $p+1$ and the model of effective diffusion applies at orders lower or equal to $p$. When the fluid is in motion and at $\mathbb{P e}=\mathcal{O}(\varepsilon)$, the transport regime is advective-diffusive and low scale separation induces an apparent regime of 
dispersion. Therefore, predominant diffusion and advection-diffusion in a porous medium at low scale separation lead to apparent regimes of advection-diffusion and dispersion, respectively. We may therefore conclude that scale separation is a crucial issue whenever the fluid is in motion, since low scale separation then induces modification of the apparent transport regime, while it remains of diffusive type at the second order when the fluid is at rest.

\section{A. ANTI-SYMMETRY OF TENSOR $E_{i j k}$}

\section{WITH RESPECT TO ITS FIRST TWO INDICES}

Let $\alpha$ be a test function satisfying the condition of having zero mean. The variational formulation associated with $\chi_{j}$ is

$$
\int_{\Omega_{\mathrm{p}}} D_{0} \frac{\partial \alpha}{\partial y_{i}} \frac{\partial \chi_{j}}{\partial y_{i}} d \Omega=-\int_{\Omega_{\mathrm{p}}} D_{0} \frac{\partial \alpha}{\partial y_{j}} d \Omega,
$$

and the variational formulation associated with $n_{j k}$ reads

$$
\left\{\begin{array}{l}
\int_{\Omega_{\mathrm{p}}} \frac{\partial \alpha}{\partial y_{i}} D_{0}\left(\frac{\partial n_{l m}}{\partial y_{i}}+\chi_{m} \delta_{i l}\right) d \Omega= \\
\int_{\Omega_{\mathrm{p}}} \alpha D_{0}\left(\frac{\partial \chi_{m}}{\partial y_{l}}+\delta_{l m}\right) d \Omega .
\end{array}\right.
$$

By taking $\alpha=\eta_{l m}$ in Eq. 60 and $\alpha=\chi_{j}$ in Eq. 61), we deduce

$$
\left\{\begin{array}{l}
\int_{\Omega_{\mathrm{p}}} D_{0}\left(\frac{\partial \eta_{l m}}{\partial y_{j}}+\chi_{j} I_{l m}\right) d \Omega=\int_{\Omega_{\mathrm{p}}} D_{0} \chi_{m} \frac{\partial \chi_{j}}{\partial y_{l}} \\
-\int_{\Omega_{\mathrm{p}}} D_{0} \chi_{j} \frac{\partial \chi_{m}}{\partial y_{l}} d \Omega .
\end{array}\right.
$$

The left hand side of the above equation is actually the definition of $E_{j l m}$. Using Eq. (62) for determining the expression of $E_{j l m}$, we get: $E_{j l m}=E_{j l m}$. Since the medium is macroscopically homogeneous, $E_{i j k}$ does not depend on the macroscopic variable $\vec{x}$. Consequently, the anti-symmetry with respect the first two indices implies that

$$
\frac{\partial}{\partial x_{i}}\left(E_{i j k} \frac{\partial^{2} c^{0}}{\partial x_{j} \partial x_{k}}\right)=0 .
$$

\section{B. Boundary VAlue PROBLEM FOR $c^{2}$ WHEN $\mathbb{P} e=\mathcal{O}(\varepsilon)$}

Firstly, using Eq. (43), and the second order of Eq. (40)

$$
\frac{\partial v_{i}^{1}}{\partial y_{i}}+\frac{\partial v_{i}^{0}}{\partial x_{i}}=0
$$

while bearing Eq. (12) in mind, we obtain

$$
\left\{\begin{aligned}
\frac{\partial}{\partial y_{i}}\left(c^{0} v_{i}^{1}\right) & =-c^{0} \frac{\partial v_{i}^{0}}{\partial x_{i}} \\
\frac{\partial}{\partial y_{i}}\left(c^{1} v_{i}^{0}\right) & =v_{i}^{0} \frac{\partial c^{1}}{\partial y_{i}} .
\end{aligned}\right.
$$

Next, we use Eq. (50) and Eq. (16) to derive the expressions of $\partial c^{0} / \partial t$ and of $\partial c / \partial x_{i}$, respectively. Finally, substituting the thus derived expressions into Eqs. (49), while using Eq. (21), we obtain Eq. (52).

\section{REFERENCES}

Auriault J.-L. and Adler P.: Taylor dispersion in porous media: Analysis by multiple scale expansions, Advances in Water Resources, 18, (4), 217-226 (1995).

Auriault J.-L., Geindreau C. and Boutin C.: Filtration law in porous media with poor separation of scales, Transport in Porous Media, 60, 89-108, (2005).

Bensoussan A., Lions J.-L. and Papanicolaou G.: Asymptotic Analysis for Periodic Structures, North-Holland Publishing Company, Amsterdam, (1978).

Sanchez-Palencia, E.: Non-Homogeneous Media and Vibration Theory, Lecture Notes in Physics 127, Springer-Verlag, Berlin (1980). 